\title{
Lifetime enhancement for clustering protocols in heterogeneous wireless sensor networks
}

\author{
Basim Abood ${ }^{1}$, Yasser Kareem Al-Rikabi ${ }^{2}$ \\ ${ }^{1}$ University of Sumer, College of Computer Science and Information Technology, Iraq \\ ${ }^{2}$ University of Sumer, Center of Computer, Iraq
}

\begin{tabular}{l} 
Article Info \\
\hline Article history: \\
Received Sep 17, 2018 \\
Revised Nov 18, 2018 \\
Accepted Jan 27, 2019 \\
\hline Keywords: \\
Fuzzy logic \\
Heterogeneous wireless sensor \\
networks \\
Network lifetime \\
Particle swarm optimization \\
(PSO) \\
Stable election protocol \\
clustering
\end{tabular}

\begin{abstract}
In this paper, we propose a new clustering method called fuzzy stable election protocol (FSEP), which is capable to overcome the bottleneck problem and addressing the uneven energy consumption problem in heterogeneous WSNs. We also propose an energy-efficient routing method called particle swarm optimization routing method (PSORM) to find the optimal routing path for the heterogeneous WSNs. PSORM seeks to investigate the problems of balancing energy consumption and maximization of network lifetime. To demonstrate the effectiveness of FSEP-PSORM in terms of lessening end-to-end delay, balancing energy consumption, and maximization of heterogeneous network lifetime, we compare our method with three approaches namely, chessboard clustering approach, PEGASIS, and LEACH. Simulation results show that the network lifetime achieved by FSEP-PSORM could be increased by nearly 38\%, 45\%, and $60 \%$ more than that obtained by PEGASIS, LEACH and stable election protocol clustering (SEP), respectively.
\end{abstract}

Copyright $\odot 2019$ Institute of Advanced Engineering and Science. All rights reserved.

\section{Corresponding Author:}

Basim Abood,

College of Computer Science and Information Technology,

University of Sumer, Iraq.

Email: bas.eng1984@gmail.com

\section{INTRODUCTION}

A Wireless sensor network (WSN) usually consists of a base station (BS) whose role is to communicate with a number of wireless sensor nodes via a radio link. As the nodes in WSNs are battery operated, and it may not be feasible some time to replace battery, so energy consumption is one of the major issues that needs special attention. So there is a requirement of efficient mechanisms for energy consumption for various operations such as data aggregation, computation, or communication in WSN [1].

Broadly, there are two types of environments in WSNs defined as follows: homogeneous and heterogeneous. Many protocols have been defined for homogeneous environment, but the performance of these protocols is not found good in heterogeneous environment. The existing methods for prolonging the network lifetime focus on homogeneous WSNs, where all the nodes in the network are of the same type. However, the continued advances in miniaturization of processors and in low power communications combined with mass-produced sensors have enabled the development of a wide variety of nodes. When more than one type of nodes is integrated into a WSN, it is called heterogeneous WSN. Many of the existing civilian and military applications heterogeneous WSNs do not differ substantially from their homogeneous counterparts [2]. In a heterogeneous sensor network, the basic sensors (L-sensors) are simple and perform the sensing task, whereas some other nodes, often called the cluster heads (H-sensors), are more powerful and focus on communications and computations. An example of a heterogeneous sensor network is shown in Figure 1. 


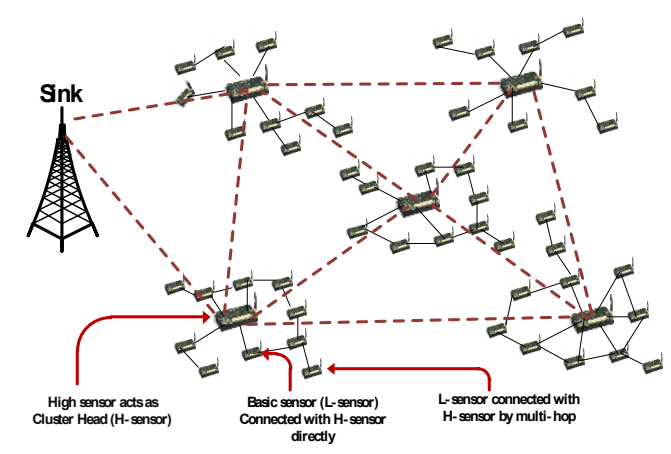

Figure 1. A heterogeneous sensor network structure

In a WSN is to divide the network into a number of clusters where a cluster head serves as a fusion point for the aggregation of all the data gathered in a cluster. As a result, the amount of data that is actually transmitted from the cluster to the sink is reduced, which consumes less resources. The clustering approaches have also proposed to address the uneven energy consumption (UEC) problem in WSNs [3]. In typical sensor networks, the many-to-one traffic pattern is dominant, that is, a large number of sensor nodes send data to the sink. Thus, sensor nodes near the sink have much heavier traffic burden and run out of power much faster than other nodes. The short lifetime of these critical nodes dramatically reduces sensor network lifetime. However, there is another UEC problem in schemes with fixed cluster heads. The nodes near the cluster head are referred to as critical nodes. Every transmission of a node in the cluster to the cluster head has to go through one of these critical nodes. This is because the critical nodes are the last hop nodes for all the paths. Hence, among all the nodes in a cluster, the critical nodes have the highest burden of relaying data. Because the critical nodes have much heavier traffic load than other nodes in a cluster, they will run out of their power much faster than other nodes. When the critical nodes drain out their energy and become unavailable, other nodes will not be able to send packets to the cluster head, and the entire cluster becomes unavailable even though the remaining energy in many sensor nodes is still high. The remaining energy in the peripheral nodes is wasted.

Recent studies have shown the heterogeneous sensor networks inherent advantages in terms of reliability, robustness, and energy efficiency [4]. A growing trend of heterogeneous designs has also been witnessed in a number of applications [5-6]. To prolong the lifetime of heterogeneous WSNs, novel device placement methods [7], routing protocols [8-9], and topology management strategies [10] have been introduced. The authors [11] proposed the energy-efficient heterogeneous clustered (EEHC) scheme for WSNs to increase the energy efficiency, stability, and lifetime of the network. EEHC considered three types of nodes. EEHC considered heterogeneity of sensor nodes based on the weighted probability of election of CHs. This protocol gives better performance as compared with LEACH and SEP.

In this paper, we propose using the cluster method to organize the heterogeneous sensor nodes under the concept of stable election protocol clustering. The main issues of this method are letting the cluster head knows which sensors are in its cluster and letting the sensors know to which cluster they belong. Afterward, we propose a novel clustering method called fuzzy stable election protocol (FSEP) to overcome the bottleneck problem and addressing the UEC problem in heterogeneous WSNs. On the other side, we propose an energy-efficient routing method called particle swarm optimization routing method (PSORM) to find the optimal routing path for the heterogeneous WSNs, in both intra-cluster and inter-cluster. FSEP-PSORM is therefore proposed for heterogeneous WSNs to address UEC problem, balancing energy consumption, and maximization of network lifetime.

The rest of this paper is organized as follows. The proposed method FSEP-PSORM is presented in Section 2. Section 3 describes the Performance evaluation. Finally, conclusion and discussion are presented in Section 4.

\section{FUZZY STABLE ELECTION PROTOCOL AND PARTICLE SWARM OPTIMIZATION ROUTING METHOD}

For the proposed model, any sensor node (H-sensor/L-sensor) runs out of energy, communication links between various sensor nodes and the base station will break. This is considered as the end of the network lifetime. Because the lifetime of each sensor node depends on energy consumption, it is important to 
preserve residual energy of these nodes in such a way that overall network lifetime is extended. The primary goal of this paper is to design a new protocol that will prolong the lifetime of the heterogeneous WSNs through limiting energy cost as well as equal distribution of energy consumption. The new protocol is capable to overcome the bottleneck problem and addressing the UEC problem in heterogeneous WSNs. To achieve this protocol, we will treat two issues. First, we seek to use fuzzy logic [12-13] to enhance the performance of the stable election protocol clustering. The new clustering called FSEP, which is used fuzzy logic to select the best cluster heads; Later, we describe the proposed routing method, which exploits the PSO [14-16] to find the optimal routing path from the source L-sensor to the H-sensor and from a $\mathrm{H}$ sensor to the sink.

The proposed protocol assumes that (i) all L-sensors have the same maximum transmission range and the same amount of initial energy, (ii) each L-sensor is aware of its location as well as that of its neighbors and the cluster heads it belongs, (iii) all $\mathrm{H}$-sensors have the same maximum transmission range and the same amount of initial energy, and (iv) each H-sensor aware of its location as well as that of its neighbors and the sink.

\subsection{Stable Election Protocol (SEP) \\ 2.1.1 Network Model}

In this section, we describe the SEP protocol. Assume that there are $\mathrm{N}$ sensor nodes. Nodes always have data to transmit to a base station, which is often far away from the sensing area. The network is organized into a clustered hierarchy where every cluster has a $\mathrm{CH}$, responsible for executing fusion function to reduce correlated data produced by the sensor nodes within the same cluster. The CHs directly transmit the aggregated data to the base station. We suppose that the nodes are stationary. SEP does not require energy knowledge sharing but is based on assigning weighted election probabilities of each node to become a $\mathrm{CH}$ according to their respective energy. By using this approach, SEP ensures that the $\mathrm{CH}$ is randomly selected based on the fraction of energy of each node. This also results in a uniform distribution of energy consumption. In SEP, the election probabilities are weighted by the initial energy of a node relative to that of other nodes in the network. This prolongs the time interval before the death of the first node (stability period), which is crucial for many applications where the feedback from the sensor network must be reliable. In SEP, $\mathrm{H}$ sensor becomes CH more often than L sensor. Let $\boldsymbol{E}_{\mathbf{0}}$ be the initial energy of $\boldsymbol{L}$ sensors, and $\boldsymbol{m}$ be the fraction of $H$ sensors, which own $\alpha$ times more energy than the normal ones. Thus, there are $\boldsymbol{m} \boldsymbol{N}$ to $H$ sensors equipped with an initial energy of $(\mathbf{1}-\boldsymbol{\alpha}) \boldsymbol{E}_{\mathbf{0}}$; and $(\mathbf{1}-\boldsymbol{m}) \boldsymbol{N}$, (L sensors) with an initial energy of $\boldsymbol{E}_{\mathbf{0}}$. Thus, the total initial energy of the two level heterogeneous networks is:

$$
\text { Etotal }=N(1-m) E_{0}+m N E_{0}(1+\alpha) N E_{0}(1+\alpha m)
$$

So, the total energy of the system is increased by a factor of $(\mathbf{1}+\boldsymbol{\alpha m})$.

Let $\boldsymbol{P}_{\boldsymbol{a d} \boldsymbol{v}}$ be the weighted election probability of advance nodes. Optimum probability $\left(\boldsymbol{P}_{\text {opt }}\right)$ of each node to become $\mathrm{CH}$ can be calculated by (2).

$$
P_{a d v}=\frac{P_{o p t}}{1+\alpha m} \times(1+\alpha)
$$

The threshold is given by (3).

$$
T(s)=\left\{\begin{array}{c}
\frac{P_{a d v}}{1-P_{a d v}\left[r \cdot \bmod \frac{1}{P_{a d v}}\right]} \text { if } s \in G \\
0 \text { otherwise }
\end{array}\right.
$$

In this paper, we consider a sensor network consisting of $\mathrm{N}$ sensor nodes deployed over a vast field to continuously monitor the environment.

\subsubsection{Energy Consumption Model}

For the realistic, the first order radio model that will be used in LEACH [17], as a communication model between sensor nodes. Both the free space ( $\boldsymbol{d}^{2}$ power loss) and the multipath fading ( $\boldsymbol{d}^{\mathbf{4}}$ power loss) channel models are used, depending on the distance between the transmitter and receiver. The energy consumption in transmitting a packet with $\boldsymbol{k}$-bits over distance $\boldsymbol{d}$. $\boldsymbol{E}_{\text {elec }}$ is the amount of energy consumption per bit to run the transmitter or receiver circuitry. $\boldsymbol{E}_{\boldsymbol{f} s}$, and $\boldsymbol{E}_{\boldsymbol{m} \boldsymbol{p}}$ is the amount of energy per bit dissipated in the RF amplifier according to the distance $\boldsymbol{d}_{\mathbf{0}}$ which can be obtained from (4), and (5).

\footnotetext{
Lifetime enhancement for clustering protocols in heterogeneous wireless sensor networks (Basim Abood)
} 


$$
\begin{aligned}
& E_{n} T(k)=\left\{\begin{array}{l}
k \times\left(E_{\text {elec }}+E_{f s} \times d^{2}\right), \text { if } d<d_{0} \\
k \times\left(E_{\text {elec }}+E_{m p} \times d^{4}\right), \text { if } d \geq d_{0}
\end{array}\right. \\
& d_{0}=\sqrt{\frac{E_{f s}}{E_{m p}}}
\end{aligned}
$$

The amount of energy consumption in receiving a packet with $k$ bits can be calculated by 6 .

$$
E_{n} R(k)=k \times E_{\text {elec }}
$$

The radio energy model parameters present details in Table 1. Fuzzy Clustering System Architecture as shown in Figure 2.

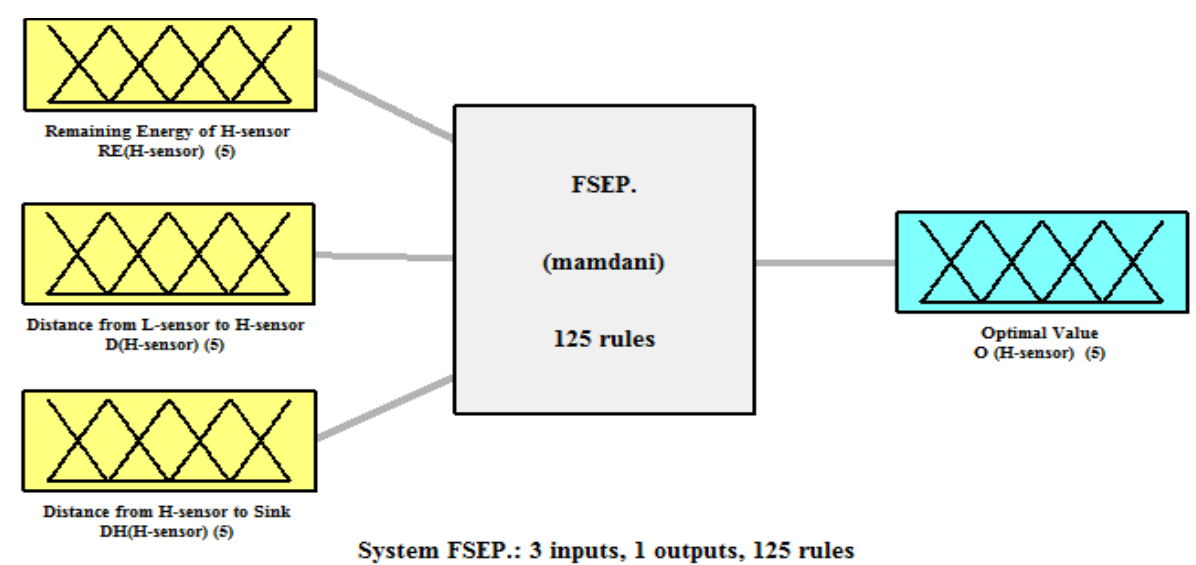

Figure 2. Fuzzy Clustering System Architecture

Table 1. Parameters of the First Radio Model for L-Sensors and H-Sensors

\begin{tabular}{ccc}
\hline \multicolumn{2}{c}{ Parameter } & value \\
\hline \multirow{3}{*}{ L- sensor } & $E_{\text {elec }}$ & $50 \mathrm{~nJ} / \mathrm{bit}$ \\
& $E_{f s}$ & $10 \mathrm{pJ} / \mathrm{bit} / \mathrm{m}^{2}$ \\
& $E_{\text {amp }}$ & $100 \mathrm{pJ} / \mathrm{bit} / \mathrm{m}^{2}$ \\
\hline \multirow{3}{*}{ H- sensor } & $E_{\text {elec }}$ & $100 \mathrm{~nJ} / \mathrm{bit}$ \\
& $E_{f s}$ & $10 \mathrm{pJ} / \mathrm{bit} / \mathrm{m}^{2}$ \\
& $E_{\text {amp }}$ & $200 \mathrm{pJ} / \mathrm{bit} / \mathrm{m}^{2}$ \\
\hline
\end{tabular}

\subsection{Fuzzy Clustering Approach}

As we introduced in the previous section, each L-sensor belongs to clusters. In Fuzzy Clustering model, when an L-sensor detects an event and wants to transmit its packets, it selects a best cluster head (H-sensor). To achieve this, we make use of fuzzy logic. The objective of fuzzy logic is therefore to calculate the optimal value of the best cluster head $\mathrm{O}$ (H-sensor) that depended on the remaining energy of $\mathrm{H}$-sensor $\mathrm{RE}$ (H-sensor), the distance from L-sensor to $\mathrm{H}$-sensor D (H-sensor), and the distance from the $\mathrm{H}$-sensor to the sink DH (H-sensor). Figure 2, show the fuzzy logic with three input variables (RE, normalize D, and normalize DH), and an output, with universal of discourse $[0 \ldots .10],[0 \ldots .1],[0 \ldots .1]$, and $[0 . .1]$, respectively. Fuzzy Clustering uses five membership functions for each input and an output variable, as shown in Figure 3.

In Fuzzy Clustering, the fuzzified values are processed by the inference engine, which consists of a rule base and various methods to infer the rules. The Tables 2-6 show the IF-THEN rules used in Fuzzy Clustering, with a total number of $5^{3}=125$ for the fuzzy rule base. As example, IF RE is Very High and D is Very Near and DH is Very Near THEN O is Very Good. All these rules are processed in a parallel manner by a fuzzy inference engine. At the end, the defuzzification finds a single crisp output value from the solution fuzzy space. This value represents the node cost. Practice defuzzification is carried out using center of gravity method given by 7 [13]: 


$$
O(H-\text { sensor })=\sum_{k=1}^{n} U_{k} * c_{k} / \sum_{k=1}^{n} U_{k}
$$

Where $U_{k}$ is the output of rule base $k$, and $c_{k}$ is the center of the output membership function for $n$ rule base number.
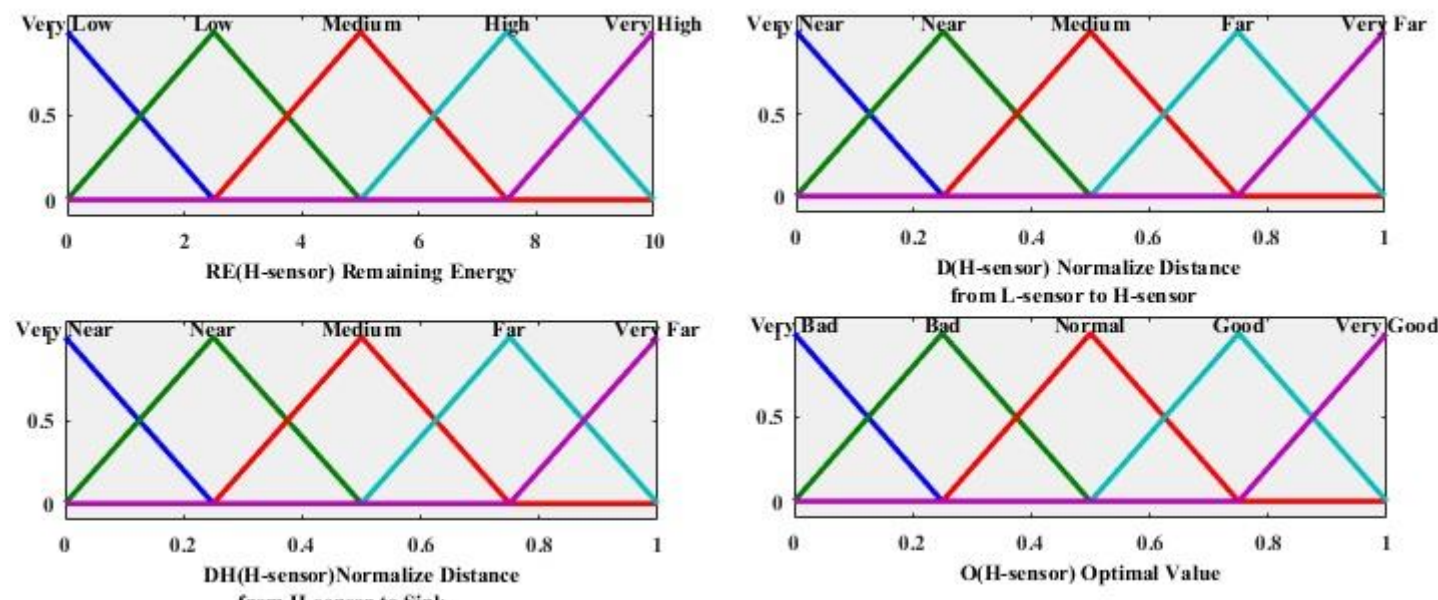

Figure 3. Membership graph for three inputs (RE, normalize distance from L-sensor to H-sensor, and normalize distance from $\mathrm{H}$-sensor to sink) and the output (optimal value)

Table 2. IF-THEN Rules, Where Energy is Very Low

\begin{tabular}{cccccc} 
DH & V. Near & Near & Medium & Far & V. Far \\
\hline V. Near & Normal & Bad & Bad & V. Bad & V. Bad \\
Near & Normal & Bad & V. Bad & V. Bad & V. Bad \\
Medium & Bad & V. Bad & V. Bad & V. Bad & V. Bad \\
Far & Bad & V. Bad & V. Bad & V. Bad & V. Bad \\
V. Far & V. Bad & V. Bad & V. Bad & V. Bad & V. Bad \\
\hline
\end{tabular}

Table 3. IF-THEN Rules, Where Energy is Low

\begin{tabular}{cccccc} 
DH & V. Near & Near & Medium & Far & V. Far \\
\hline V. Near & Normal & Normal & Bad & Bad & V. Bad \\
Near & Normal & Bad & V. Bad & V. Bad & V. Bad \\
Medium & Normal & Bad & V. Bad & V. Bad & V. Bad \\
Far & Bad & V. Bad & V. Bad & V. Bad & V. Bad \\
V. Far & V. Bad & V. Bad & V. Bad & V. Bad & V. Bad \\
\hline
\end{tabular}

Table 4. IF-THEN Rules, Where Energy is Medium

\begin{tabular}{cccccc} 
DH & V. Near & Near & Medium & Far & V. Far \\
\hline V. Near & Good & Good & Good & Normal & Normal \\
Near & Good & Good & Normal & Normal & Bad \\
Medium & Good & Good & Normal & Bad & Bad \\
Far & Normal & Normal & Normal & Bad & V. Bad \\
V. Far & Normal & Normal & Bad & V. Bad & V. Bad \\
\hline
\end{tabular}

Table 5. IF-THEN Rules, Where Energy is High

\begin{tabular}{cccccc} 
DH & V. Near & Near & Medium & Far & V. Far \\
\hline V. Near & V. Good & V. Good & Good & Normal & Normal \\
Near & V. Good & V. Good & Good & Bad & Normal \\
Medium & V. Good & V. Good & Good & Bad & Bad \\
Far & Good & Good & Normal & V. Bad & V. Bad \\
V. Far & Normal & Normal & Normal & V. Bad & V. Bad \\
\hline
\end{tabular}

Lifetime enhancement for clustering protocols in heterogeneous wireless sensor networks (Basim Abood) 


\begin{tabular}{cccccc}
\multicolumn{6}{c}{ Table 6. IF-THEN Rules, Where Energy is Very High } \\
\hline DH & V. Near & Near & Medium & Far & V. Far \\
\hline V. Near & V. Good & V. Good & V. Good & Normal & Normal \\
Near & V. Good & V. Good & V. Good & Normal & Normal \\
Medium & V. Good & V. Good & Good & Normal & Bad \\
Far & $\begin{array}{l}\text { V. Good } \\
\text { Good }\end{array}$ & Good & Bad & V. Bad \\
V. Far & Good & Good & Good & Bad & V. Bad \\
\hline
\end{tabular}

\subsection{Particle Swarm Optimization Routing Method}

Unbalanced energy consumption is an inherent problem in WSNs characterized by the multi-hop routing and many-to-one traffic pattern. This uneven energy dissipation can significantly reduce network lifetime. Generally, in routing algorithm, the best path is chosen for transmission of data from source to destination. Over a period, if the same path is chosen for all communications to achieve battery performance in terms of quick transmission time, then those nodes on this path will drain fast. In this paper, we therefore exploit the PSO algorithm to find the optimal routing path for the heterogeneous WSNs. PSORM seeks to investigate the problems of balancing energy consumption and maximization of network lifetime.

After selecting the best cluster head, PSORM seeks to find the optimal routing path from the source basic node to the sink through the H-sensor. It selects the best node from candidate nodes (neighbors) in the forwarding paths in both (i) by favoring the highest remaining energy for L-sensor and the short distance (minimum number of hops) to the $\mathrm{H}$-sensor and (ii) by favoring the highest remaining energy for $\mathrm{H}$-sensor and the short distance to the sink.

PSORM considers a tree structure in terms of $(\boldsymbol{S}, \boldsymbol{F})$, where $\boldsymbol{S}$ is the set of candidate nodes in the forwarding path and $F$ is the fitness function, which assigns a fitness value $f(s)$ to each candidate node $s \in S$. The fitness value $f(s)$ is determined depending on the remaining energy of node $s$ and the distance from node $s$ to the sink. The tree node is explored on the basis of its fitness value. Where the candidate node that has the highest remaining energy and the lowest distance to the sink will be selected as the best node in the forwarding path to the sink. The fitness function $f(s)$ we used is given by 8 .

$$
f(s)=\propto * R E(s)+\frac{1}{\beta * M H(s)}
$$

where $\boldsymbol{R} \boldsymbol{E}(\boldsymbol{s})$ is the remaining energy for node $\boldsymbol{s}, \boldsymbol{M H}(\boldsymbol{s})$ is the distance from node s (minimum number of hops) to the destination, and $\alpha, \boldsymbol{\beta}$ are constant values ( $\alpha=\mathbf{0 . 1}$ and $\boldsymbol{\beta}=\mathbf{0 . 1 6}$ ). In PSORM, the candidate nodes are selected as the next hops (neighbors) of the source where each employed swarm in PSO is nominated to one of these nodes because each employed swarm represents to a candidate node in the following path. All employed swarms compute the fitness functions of their nodes and share this information with neighbors. The flow chart of the proposed method FSEP-PSORM is shown in Figure 4.

\section{PERFORMANCE EVALUATION}

To demonstrate the effectiveness of FSEP-PSORM in terms of balancing energy consumption and maximizing heterogeneous network lifetime, simulation results of theproposed method compared with those of two well-known clustering protocols that are designed for homogeneous WSNs, that is, LEACH [17] and PEGASIS[18].

\subsection{Simulation setup}

Simulations are carried out in MATLAB R2011a (version 7.12.0), For our proposed method, $200 \mathrm{~L}$ sensors and $10 \mathrm{H}-$ sensors are randomly deployed in topographical area of dimension $100 \mathrm{~m} \times 100 \mathrm{~m}$. $\mathrm{H}$-sensors are installed in the topographical area under the cluster scheme; Whereas L-sensors are distributed around $\mathrm{H}$-sensors used the cluster algorithm. On the other hand, LEACH and PEGASIS are designed for homogenous sensor networks; for fair comparison, another set of $150 \mathrm{~L}$-sensors are randomly deployed in a topographical area of the same dimension. Here, we consider that higher additional L-sensors are used in LEACH and PEGASIS to make the investment similar. Of course, the actual costs of $\mathrm{H}$-sensor and L-sensor depend on the type of sensor, manufacture, and so on, and this issue is out of the scope of this paper. The simulation runs for 2000 transmission packets (rounds). An L-sensor in our proposed generates three data packets per round, and an L-sensor in LEACH and PEGASIS generates two packets per round. Thus, the total volume of data generated in our method is the same as in LEACH and PEGASIS. 
All the control data packets have the same length equal to 2 Kbytes. In the topographical area, there is data sink which located at $(90 \mathrm{~m}, 90 \mathrm{~m})$. All L-sensors and H-sensors have the same initial energy 0.5 and $2.5 \mathrm{~J}$ with a sensed transmission of 20 and $80 \mathrm{~m}$, respectively. The approaches are carried out using the first radio model that is largely used in the area of routing protocol evaluation in WSNs [21].

\subsection{Simulation results}

In FSEP-PSORM, energy consumption among all nodes (L-sensors and H-sensors) in the network is balanced in expectation that all nodes should run out of energy at nearly the same time. In this section, the simulations are focused on evaluating the performance of FSEP-PSORM in terms of lessening end-to-end delay, balancing energy consumption and maximization of network lifetime by comparing with LEACH, and PEGASIS. Table 7 presents the simulation parameters details.

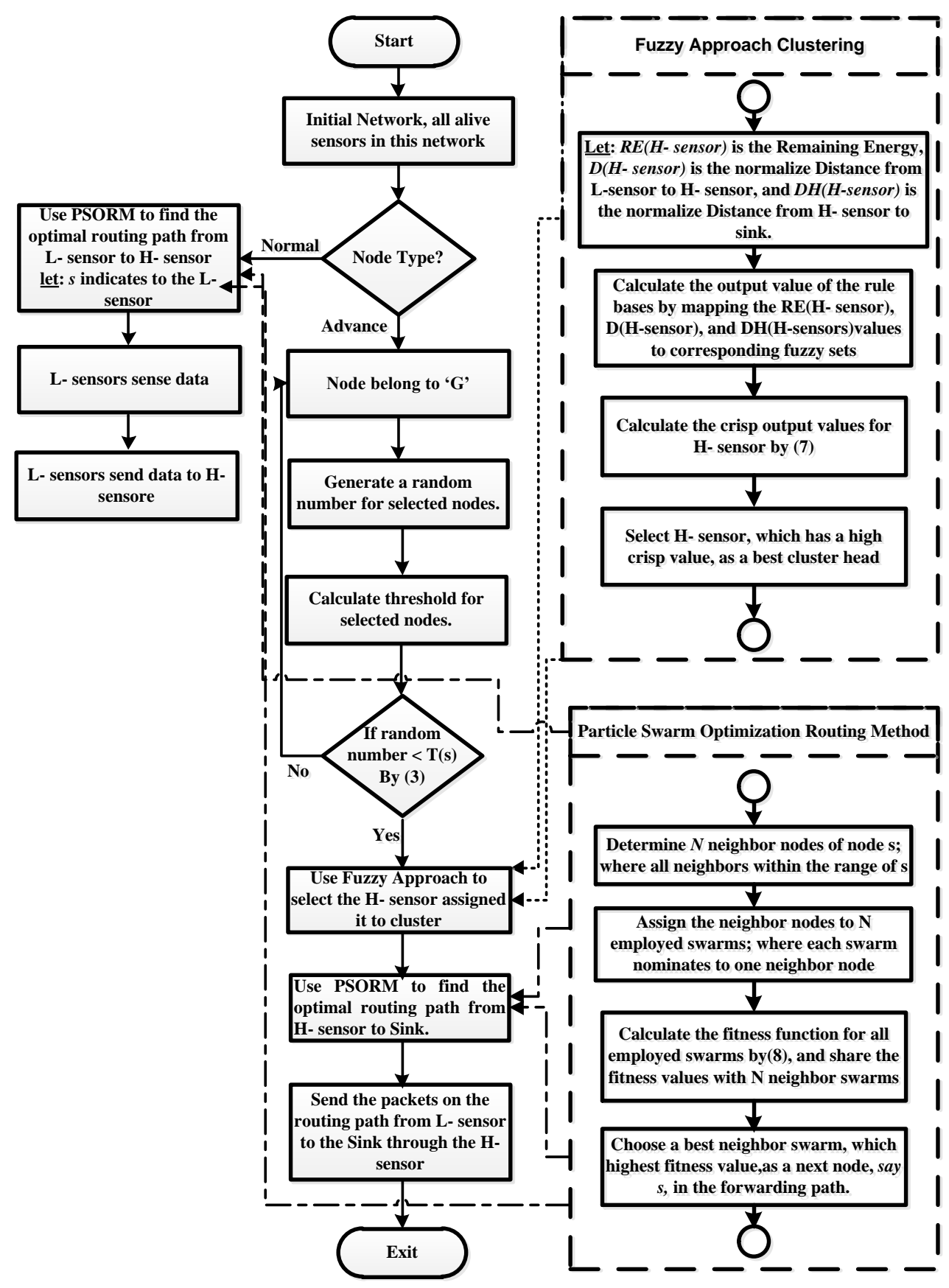

Figure 4. Flow chart of fuzzy stable election clustering-particle swarm optimization routing method (FSEP-PSORM) 
Table 7. Simulation Parameters

\begin{tabular}{|c|c|}
\hline Parameter & Value \\
\hline Topographical Area (meters) & $(100 \mathrm{~m} \times 100 \mathrm{~m})$ \\
\hline Sink location (meters) & $(90 \mathrm{~m} \times 90 \mathrm{~m})$ \\
\hline $\begin{array}{l}\text { No. in FSEP- } \\
\text { PSORM }\end{array}$ & 200 \\
\hline $\begin{array}{l}\text { No. in LEACH and } \\
\text { PEGASIS }\end{array}$ & 150 \\
\hline $\begin{array}{l}\text { Limit of } \\
\text { transmission } \\
\text { distance }\end{array}$ & $20 \mathrm{~m}$ \\
\hline Initial energy & $0.5 \mathrm{~J}$ \\
\hline $\begin{array}{l}\text { No. in FSEP- } \\
\text { PSORM }\end{array}$ & 10 \\
\hline $\begin{array}{l}\text { Limit of } \\
\text { transmission } \\
\text { distance }\end{array}$ & $80 \mathrm{~m}$ \\
\hline Initial energy & $2.5 \mathrm{~J}$ \\
\hline Packet data size & $4 \times 10^{3}$ bits \\
\hline $\begin{array}{l}\text { No. of MFs (in each input and output } \\
\text { variable) }\end{array}$ & 5 \\
\hline No. of IF-THEN rules & 125 \\
\hline No. of transmission packets (rounds) & $2 \times 10^{3}$ \\
\hline
\end{tabular}

Firstly, we compare the network lifetime for the three different approachesin. Network lifetime can be defined as the time elapsed until the first node (or the last node) in the network depletes energy (dies). For the fixed routing area, the ratio of live nodes as a function of rounds (transmission packets) by using the approaches for L-sensors are shown in Figure 5(a). It can be seen that the proposed method outperforms other approaches, and also the number of live nodes of the proposed method is always higher than that of others. The network lifetime achieved by the proposed method increased by nearly $38 \%$, and $52 \%$ than that obtained by PEGASIS, and LEACH, when all packets are sent in area. For H-sensor, the network lifetime achieved by the proposed method increased by nearly $60 \%$, than that obtained by SEP, when all packets are sent in the same area as shown in Figure. 5(b)

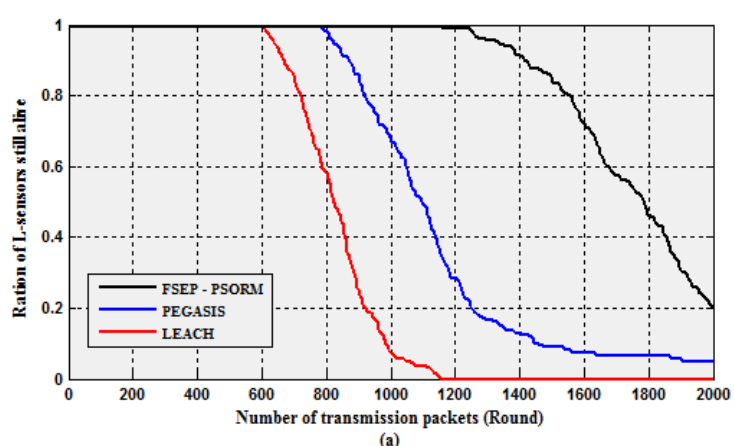

(a)

Figure 5. (a) Ratio of L-sensors still alive on different approaches (LEACH, PEGASIS, and proposed)

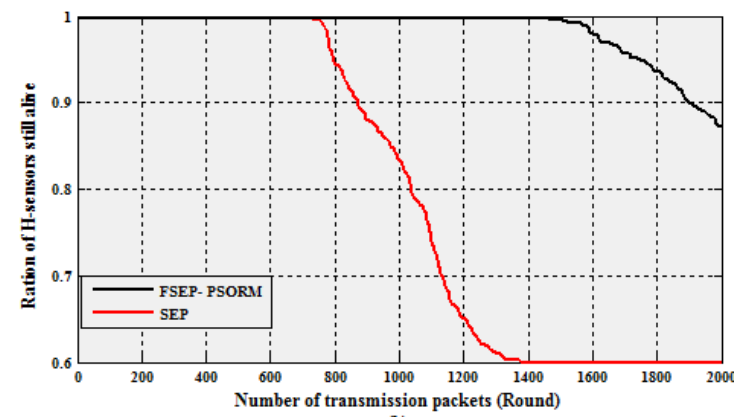

(b)

Figure 5. (b) Ratio of H-sensors still alive on two approaches (SEP, and proposed)

From Figures ( 5 a, and 5 b), and Table 8, it is clear that the proposed method outperforms other approaches in terms of balancing energy consumption and maximization of network lifetime. Figure 6(a) show the ratio remaining energy of L-sensor nodes as a function of transmission rounds based on the three approaches

Table 8. Number of Rounds with the First Dead Node Based on the Four Approaches

\begin{tabular}{ccccc}
\hline Approaches & LEACH & PEGASIS & SEP & FSEP-PSORM \\
\hline Lifetime of the first dead L-sensor (Round) & 608 & 796 & $\overline{784}$ & 1163 \\
Lifetime of the first dead H-sensor (Round) & - & - &
\end{tabular}


As the round number increases, the proposed method performs better than other approaches. As presented before, H-sensors have more initial energy than L-sensors, and also H-sensors consume more energy than L-sensors for transmitting and receiving one bit data. Figure. 6 (b) show the ratio remaining energy of H-sensor nodes as a function of transmission rounds based on the proposed and SEP approach. From Figures 6 (a) and 6 (b), it is clear that awareness energy consumption and maximization of network lifetime are achieved by the proposed method better than other approaches.

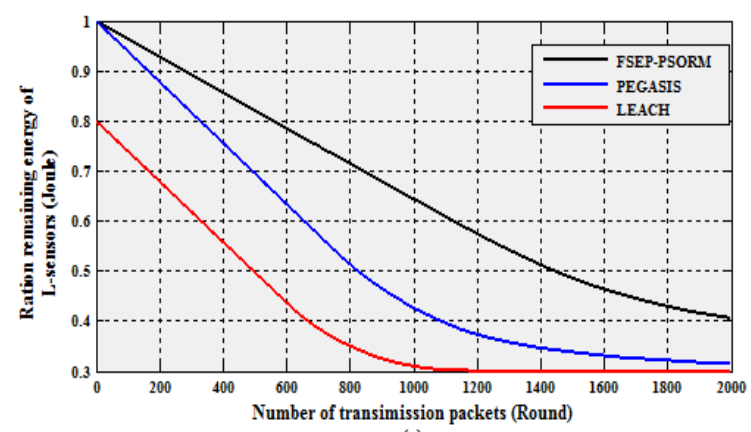

(a)

Figure 6. (a) Ratio remaining energy of L-sensors based on different approaches (LEACH, PEGASIS, and proposed)

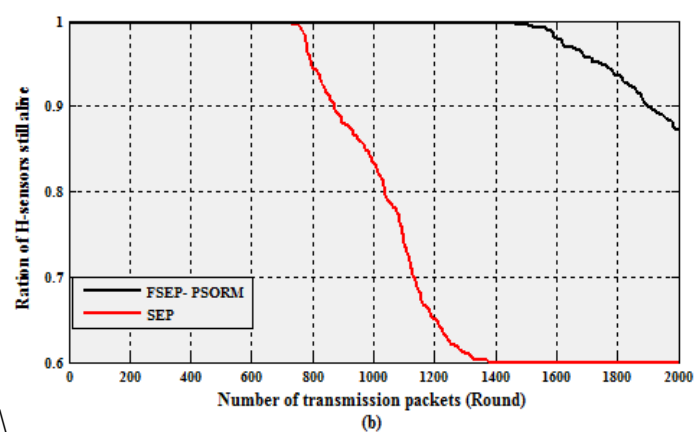

Figure 6. (b) Ratio remaining energy of H-sensors based on the two approaches (SEP, and proposed)

The delay incurred in transmission of data packets for L-sensor is also a key parameter for certain applications. The simulation time comparison among the three approaches is shown in Figure. 7(a). Clearly, it can be seen that the proposed method has shortest time delay compared with other approaches. Moreover in Figure. 7(b). The simulation time comparison for H-sensor between two approaches. It can be seen that the proposed method has shortest time delay compared with SEP approach.

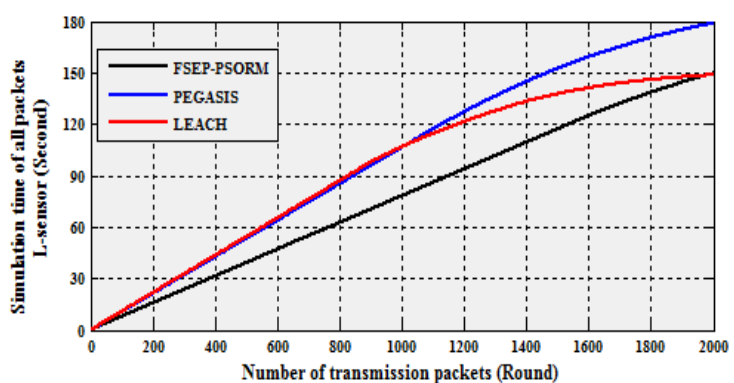

(a)

Figure 7. (a) Data transmission delay For L-sensor based on different approaches (LEACH, PEGASIS, and proposed

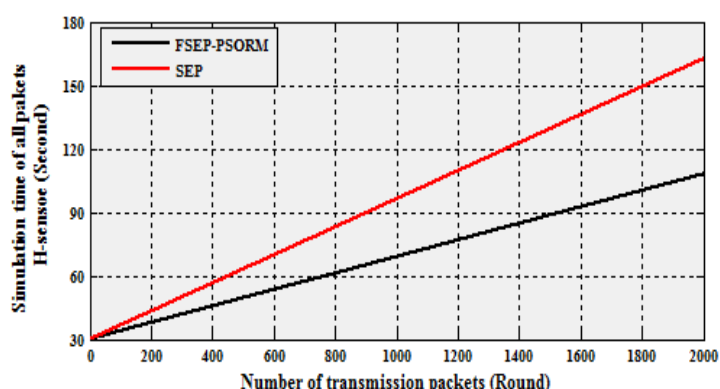

(b)

Figure 7. (b) Data transmission delay For H-sensor based on two approaches (SEP, and proposed)

Shorter time delay indicates both energy saving and efficient information transmission (especially secure and important ones). In other words, data packets are routed through different node-disjoint paths with multipath routing to avoid network congestion and prolong the network lifetime. Noted from previous simulation results, FSEP-PSORM outperforms SEP, PEGASIS, and LEACH approach in terms of lessening data transmission delay, balancing energy consumption, and maximization of network lifetime. However, some of researches on this topic are worthy of further study in the future. As a result, we have planning to exploit the mobile cluster heads to overcome the bottleneck problem and addressing the UEC in heterogeneous WSNs. The mobility could improve the balancing of energy consumption especially in the critical nodes are inside the clusters. 


\section{CONCLUSION}

Saving energy is not enough to effectively prolong the network lifetime. The UEC often results in network partition and reduce network lifetime, which deteriorates the performance. Therefore, this paper proposed an energy-efficient protocol called FSEP-PSORM for heterogeneous WSNs. First, we have used the cluster method to organize the heterogeneous sensor nodes under the concept of stable election Protocol clustering. Afterward, we have proposed a novel clustering method called FSEP to overcome the bottleneck problem and addressing the UEC problem in heterogeneous WSNs. FSEP is used fuzzy logic to determine the optimal node of cluster heads in stable election protocol clusters for WSNs. On the other side, we proposed an energy-efficient routing method called PSORM to find the optimal routing path under the concept of FSEP. Simulation results demonstrated that the proposed FSEP-PSORM makes significant improvements for heterogeneous WSNs in terms of lessening data transmission delay, balancing energy consumption, and maximization of network lifetime as compared with three well-known protocols, that is, SEP, PEGASIS, and LEACH.

\section{REFERENCES}

[1] Y. Wang, P. Shi, K. Li, and Z. Chen, "An energy efficient medium access control protocol for target tracking based on dynamic convey tree collaboration in wireless sensor networks," International Journal of Communication Systems, vol. 25, pp. 1139-1159, 2012.

[2] A. Boukerche, Algorithms and protocols for wireless sensor networks vol. 62: John Wiley \& Sons, 2008.

[3] X. Du and Y. Xiao, "Energy efficient chessboard clustering and routing in heterogeneous sensor networks," International Journal of Wireless and Mobile Computing, vol. 1, pp. 121-130, 2006.

[4] M. Yarvis, N. Kushalnagar, H. Singh, A. Rangarajan, Y. Liu, and S. Singh, "Exploiting heterogeneity in sensor networks," in INFOCOM 2005. 24th Annual Joint Conference of the IEEE Computer and Communications Societies. Proceedings IEEE, 2005, pp. 878-890.

[5] H. Wang, D. Estrin, and L. Girod, "Preprocessing in a tiered sensor network for habitat monitoring," EURASIP Journal on Advances in Signal Processing, vol. 2003, pp. 1-10, 2003.

[6] Y.-M. Huang, M.-Y. Hsieh, H.-C. Chao, S.-H. Hung, and J. H. Park, "Pervasive, secure access to a hierarchical sensor-based healthcare monitoring architecture in wireless heterogeneous networks," Selected Areas in Communications, IEEE Journal on, vol. 27, pp. 400-411, 2009.

[7] Q. Wang, K. Xu, G. Takahara, and H. Hassanein, "Transactions papers-device placement for heterogeneous wireless sensor networks: Minimum cost with lifetime constraints," Wireless Communications, IEEE Transactions on, vol. 6, pp. 2444-2453, 2007.

[8] S. Tanwar, N. Kumar, and J. W. Niu, "EEMHR: Energy-efficient multilevel heterogeneous routing protocol for wireless sensor networks," International Journal of Communication Systems, vol. 27, pp. 1289-1318, 2014.

[9] A. A. Bara'a and E. A. Khalil, "A new evolutionary based routing protocol for clustered heterogeneous wireless sensor networks," Applied Soft Computing, vol. 12, pp. 1950-1957, 2012.

[10] Y. Ma and J. H. Aylor, "System lifetime optimization for heterogeneous sensor networks with a hub-spoke technology," Mobile Computing, IEEE Transactions on, vol. 3, pp. 286-294, 2004.

[11] D. Kumar, T. C. Aseri, and R. Patel, "EEHC: Energy efficient heterogeneous clustered scheme for wireless sensor networks," Computer Communications, vol. 32, pp. 662-667, 2009.

[12] L. A. Zadeh, "Soft computing and fuzzy logic," IEEE software, vol. 11, p. 48, 1994.

[13] T. A. Runkler, "Selection of appropriate defuzzification methods using application specific properties," Fuzzy Systems, IEEE Transactions on, vol. 5, pp. 72-79, 1997.

[14] K. Bennani and D. El Ghanami, "Particle swarm optimization based clustering in wireless sensor networks: the effectiveness of distance altering," in Complex Systems (ICCS), 2012 International Conference on, 2012, pp. 1-4.

[15] R. V. Kulkarni and G. K. Venayagamoorthy, "Particle swarm optimization in wireless-sensor networks: A brief survey," Systems, Man, and Cybernetics, Part C: Applications and Reviews, IEEE Transactions on, vol. 41, pp. 262-267, 2011.

[16] N. Latiff, C. C. Tsimenidis, and B. S. Sharif, "Energy-aware clustering for wireless sensor networks using particle swarm optimization," in Personal, Indoor and Mobile Radio Communications, 2007. PIMRC 2007. IEEE 18th International Symposium on, 2007, pp. 1-5.

[17] W. B. Heinzelman, A. P. Chandrakasan, and H. Balakrishnan, "An application-specific protocol architecture for wireless microsensor networks," Wireless Communications, IEEE Transactions on, vol. 1, pp. 660-670, 2002.

[18] S. Lindsey, C. Raghavendra, and K. M. Sivalingam, "Data gathering algorithms in sensor networks using energy metrics," Parallel and Distributed Systems, IEEE Transactions on, vol. 13, pp. 924-935, 2002. 